\title{
Benefits of remote real-time side-effect monitoring systems for patients receiving cancer treatment
}

\author{
Sarah Kofoed, ${ }^{1}$ Sibilah Breen, ${ }^{1,2}$ Karla Gough, ${ }^{1}$ Sanchia Aranda1,2,3 \\ 'Department of Nursing \& Supportive Care Research, Peter MacCallum Cancer Centre, East \\ Melbourne, Victoria; ${ }^{2}$ Department of Nursing, Melbourne School of Health Sciences, University \\ of Melbourne, Victoria; ${ }^{3}$ Division of Cancer Services and Information, Cancer Institute NSW, \\ Alexandria, NSW, Australia
}

\begin{abstract}
In Australia, the incidence of cancer diagnoses is rising along with an aging population. Cancer treatments, such as chemotherapy, are increasingly being provided in the ambulatory care setting. Cancer treatments are commonly associated with distressing and serious sideeffects and patients often struggle to manage these themselves without specialized real-time support. Unlike chronic disease populations, few systems for the remote real-time monitoring of cancer patients have been reported. However, several prototype systems have been developed and have received favorable reports. This review aimed to identify and detail systems that reported statistical analyses of changes in patient clinical outcomes, health care system usage or health economic analy-
\end{abstract}

Correspondence: Sibilah Breen, Department of Nursing \& Supportive Care Research, Locked Bag 1, A'Beckett Street, Victoria, 8006, Australia.

Tel: +61.3.9656.1667 - Fax: +61.3.96563502.

E-mail: sibilah.breen@petermac.org

Key words: cancer, chemotherapy, toxicities, remote monitoring.

Acknowledgements: the authors would like to acknowledge the Victorian Cancer Agency for the provision of funding which supported the writing of this review article. We also wish to thank Ms Rose Kamateros for her invaluable proofreading skills.

Contributions: SK, SB, conception and design, analysis and interpretation of data, drafting and critical revision of article, and final approval of article; KG, $\mathrm{SA}$, analysis and interpretation of data, critical revision of article and final approval of article.

Conflict of interests: the authors have no financial conflict of interests to declare in relation to this manuscript. SB and SA currently hold two research grants with N. Kearney, the author of one of the papers critically reviewed in this manuscript.

Received for publication: 17 February 2012.

Revision received: 11 May 2012.

Accepted for publication: 28 May 2012.

This work is licensed under a Creative Commons Attribution NonCommercial 3.0 License (CC BY-NC 3.0).

CC Copyright S. Kofoed et al., 2012

Licensee PAGEPress, Italy

Oncology Reviews 2012; 6:e7

doi:10.4081/oncol.2012.e7 ses. Five papers were identified that met these criteria. There was wide variation in the design of the monitoring systems in terms of data input method, clinician alerting and response, groups of patients targeted and clinical outcomes measured. The majority of studies had significant methodological weaknesses. These included no control group comparisons, small sample sizes, poor documentation of clinical interventions or measures of adherence to the monitoring systems. In spite of the limitations, promising results emerged in terms of improved clinical outcomes (e.g. pain, depression, fatigue). Health care system usage was assessed in two papers with inconsistent results. No studies included health economic analyses. The diversity in systems described, outcomes measured and methodological issues all limited between-study comparisons. Given the acceptability of remote monitoring and the promising outcomes from the few studies analyzing patient or health care system outcomes, future research is needed to rigorously trial these systems to enable greater patient support and safety in the ambulatory setting.

\section{Introduction}

\section{The burden of cancer and treatment}

In 2007 , over 108,000 new cases of cancer were diagnosed in Australia. One in 2 Australians are diagnosed by the age of $85 .{ }^{1}$ Cancer care is increasingly being provided in the ambulatory care setting with patients commonly receiving chemotherapy and radiotherapy on an outpatient basis. Chemotherapy is a core treatment for cancer. However, related toxicity can often lead to distressing and even potentially life threatening side-effects (e.g. nausea, vomiting, mucositis, diarrhea and febrile neutropenia). ${ }^{2-11}$ While some side-effects, such as fatigue and mucositis, are not in themselves life threatening, they are associated with poorer treatment compliance, impaired quality of life, and increased infections and time spent in hospital. ${ }^{12-14}$ Fatigue also has a significant impact on patient quality of life and is associated with mood disturbance. ${ }^{14}$ Radiotherapy, a second core treatment for cancer, is also associated with distressing side effects (differing according to the site of individual treatment) which may include fatigue, mucositis, and gastrointestinal dysfunctions including diarrhea. ${ }^{15-19}$ Many patients also require surgery for their disease and will often experience significant post-surgical problems, such as pain..$^{20}$ In addition to treatment-associated morbidity, many cancer patients are required to deal with the symptom burden related to the disease itself. High symptom burden in patients with cancer is also a significant risk factor for adverse psychological adjustment. ${ }^{21}$

In the ambulatory care setting, patients are required to closely monitor and manage a range of potentially diverse and complicated sideeffects, without readily available clinical support. Patients are respon- 
sible for making potentially complex decisions about when to contact the treatment team in the event of new, escalating or unexpected sideeffects. Given that many cancer treatments are highly distressing and may be immunosuppressive, timely response to side-effects is vital to optimize patient management and prevent deterioration. It is, therefore, imperative that strategies are in place to assist patients to manage and monitor their side-effects whilst receiving outpatient cancer treatments.

\section{Benefits of telemedicine in chronic disease}

Telemedicine devices that allow for the remote, real-time monitoring of symptoms may be a cost-effective strategy to optimize cancer care in the community. Devices which remotely collect and send data to a monitoring station for interpretation and action by a clinician are increasingly being used in the management of chronic diseases, such as diabetes, asthma, heart failure, chronic obstructive pulmonary disease and chronic wound management. ${ }^{22-27}$ Research has shown that remote monitoring systems in chronic diseases can lead to improved patient quality of life, symptom control and wound healing rates, reduced lower limb amputations, decreased emergency room visits and unplanned hospitalizations, fewer bed days of care, decreased nursing home admissions in the elderly and decreased overall costs to the health system. ${ }^{26-29}$ It is likely that such systems could potentially benefit cancer patients undergoing active treatment in the ambulatory setting, as previous research utilizing submission of daily pen-and-paper measures (linked to defined nursing response algorithms) in chemotherapy patients resulted in significant improvements in side-effects such as nausea and vomiting. ${ }^{30}$ The ability to monitor chemotherapy patients at a distance in real-time via remote electronic devices should, therefore, also provide patient and health system benefits.

\section{Prototype remote side-effect monitoring systems for patients with cancer}

The earliest reported remote monitoring system used a touch-pad landline phone connected to a computer controlled digitized speech program. ${ }^{31}$ Patients called into the system daily and spoke to the system by pressing keys or talking into the receiver. The prevalence of eight key chemotherapy symptoms was reported alongside more detailed information, such as fluid intake and use of medications. Pre-set response thresholds enabled clinicians to be automatically faxed and action taken when patient responses exceeded these limits. Patient compliance with system usage was $75 \%$ with the majority of patients expressing satisfaction, finding it easy to use and finding the system increased awareness of side-effects and patterns over time. However, patients noted that the system needed to include additional symptoms for monitoring.

A group of studies by Lind et al. report on the first use of mobile phone technology to transmit data from a Bluetooth digital pen/digital paper pain diary. ${ }^{32-34}$ This system aimed to improve pain control in palliative cancer patients and also kept record of the pain medications taken. Patients completed diary entries three times a day and alerts of increases in pain were transmitted to the treatment team where they were printed and action was taken by a doctor or nurse. Initially, clinicians had low expectations of the system and the ability of patients to use it and struggled with changing their usual clinical practices. However, clinicians subsequently reported an increased awareness of patient pain and increased patient participation in their care. ${ }^{33}$ While patients initially had some difficulty understanding the technology, they reported that it was generally easy to use, that it improved contact with the clinical team and that it made them feel more secure. ${ }^{34}$

Another prototype system that used personal digital assistants for real-time remote monitoring of cancer patients was known as the handheld management system..$^{35}$ This system was developed for patients with lung and colorectal cancers undergoing chemotherapy. Patients were asked to answer five symptom questions a day with high scores alerting nurses via a pager system to contact patients via phone. The system also provided tailored self-care messages to patients who reported experiencing symptoms as well as general cancer and treatment information. Study interview data from patients and nurses indicated that the system was easy to use, had the potential to improve symptom management and communication, provided reassurance and support, promoted early detection, and did not disrupt daily routines. Some technical issues around questionnaire submission may have, however, contributed to sub-optimal patient adherence.

A second system, the Advanced Symptom Management System $\left(\right.$ ASyMS $^{\odot}$ ) has several different versions for patients with cancer undergoing chemotherapy, receiving palliative treatment or for adolescents undergoing chemotherapy ${ }^{36-42}$ ASyMS $^{\odot}$ uses Smart Phone technology to measure patient temperature and common treatment sideeffects twice daily. Alerts are generated when patient questionnaire responses exceed pre-set levels. Alerts are received by a dedicated pager system and classified as either red for urgent action (relating to potentially life-threatening events) or as amber where non-urgent clinical intervention was required. Nurses responding to alerts access a secure website to view patient data reports and then contact patients to provide clinical intervention. Patient devices also provide self-care information to assist in side-effect management based upon reported symptoms, in addition to cancer and treatment information and historical side-effect profiles. Patient and nurse interviews in these studies reported that the system was easy to use, ${ }^{32-38}$ enhanced communication, ${ }^{35,37}$ increased patient reassurance and support, ${ }^{33,35,37,38}$ promoted patient understanding of treatment, ${ }^{37,38}$ assisted with symptom management, ${ }^{32-34,36}$ and promoted timely intervention. ${ }^{33,34,37}$

Weaver et al. ${ }^{43}$ also developed a mobile phone based system for patients with colon cancer receiving chemotherapy. This system also focused on temperature and common chemotherapy side-effects, and was linked to a red/amber pager alerting system alongside self-care information and historical side effect profiles. Interviews following use of this system indicated that patients and nurses were confident of using it and that patients felt less bothersome to nurses and more reassured. Two further studies ${ }^{44,45}$ have employed a device known as a Health Buddy to remotely monitor side effects in head and neck cancer patients undergoing either chemotherapy, radiotherapy or surgery. Patients answered questions daily (the number and type of questions differed with the treatment received) via a landline phone. Patients were provided with self-care advice and instructions on what they should do according to the symptoms reported. This system did not generate alerts, but rather clinicians viewed patient data daily and only contacted patients if symptoms or side-effects were escalating or unresolved. In line with other cancer remote monitoring systems, patients reported feeling reassured, more satisfied with communication and thought they had better knowledge, understanding and management of their symptoms. Patient compliance with completing questionnaires was high in both studies at $84 \%$ and $86 \%$; this was unrelated to demographic variables but positively correlated with patient quality of life.

While the initial perceptions of using remote monitoring systems in cancer have been positive, it is still imperative to understand whether the use of such systems positively and significantly impact on both patient clinical outcomes, healthcare system usage and health system costs. This review aimed to identify and evaluate studies which quantified changes in patient outcomes and/or health care system usage and costs following the implementation of remote side-effect monitoring systems for patients with cancer undergoing treatment in the ambulatory care setting. 


\section{Materials and Methods}

\section{Selection process}

Studies assessing and quantifying the outcomes of remote monitoring systems in cancer were identified by searching the literature from the period January 2000 and June 2011. The electronic databases Medline, INAHL and PsycINFO and EMBASE were searched. The following search terms were used: cancer OR (MH Neoplasms) AND phone OR (MH cellular phone) OR telecommunications OR (MH Telecommunications) $\mathrm{OR}$ remote monitoring $\mathrm{OR}$ telehealth $\mathrm{OR}(\mathrm{MH}$ Telemedicine) AND care OR (MH Ambulatory Care). In addition, we hand-searched Telemedicine and e-HEALTH and the Journal of Telemedicine and Telecare for articles not retrieved by our search strategy. Reference lists of articles retrieved were also hand-searched.

Inclusion criteria were studies that: i) remotely monitored cancer treatment side effects (i.e. in the ambulatory care setting) with the aid of a technological interface (e.g. computer; mobile phone); and ii) quantified changes in patient outcomes; or iii) quantified changes in health care system utilization; or iv) quantified changes in health system costs.

Exclusion criteria included studies of: i) phone call or video assessments initiated by a health professional or outreach calls; ii) technology used to capture symptom information immediately before an appointment or on the day of presentation for treatment; iii) centers for patients to call with symptom concerns or need for advice; iv) telemedicine facilities for communication between health professionals (e.g. multidisciplinary meetings at more than one site, second opinions, support for rural practitioners, centralized pathology review); and v) studies reporting only on the development of system prototypes and/or with no statistical analyses of pre-specified patient or health service outcomes/variables.

\section{Study variables}

The study variables used for reviewing papers included: country of origin, year of publication, study design, study hypotheses, patient and clinician population, sample size, description of the remote monitoring systems (data collection, alerting systems, clinical responses, duration and frequency of monitoring), patient and clinician adherence to use of the monitoring system, previous publication of the monitoring system, patient clinical and health system usage outcomes, health economic analyses, and any potential sources of bias. In addition the level of evidence associated with each study design was categorized using National Health and Medical Research Council of Australia guidelines. ${ }^{46}$ These categories reflect the level of bias inherent in different study designs when assessing the effectiveness of health interventions with a lower number (e.g. I or II) indicating less bias than a higher number (e.g. III or IV).

\section{Analysis}

Assessment of studies identified in relation to pre-specified study variables was undertaken independently by three authors (SK, SB and KG). These assessments were then compared and any discrepancies discussed until agreement was reached.

\section{Results}

Twenty-five studies that centered on a real-time remote symptom monitoring system in cancer patients were identified (Figure 1). Of these, five papers met the inclusion criteria including one case control study, ${ }^{47}$ one post test case series ${ }^{48}$ and three randomized controlled tri- als (RCT) ${ }^{49-51}$ The systems examined in these studies have the common basis of employing a remote device (such as a Smart Phone or automated interactive telephone calls) to facilitate the generation of alerts when symptoms or side-effects reach a pre-defined threshold of severity. These alerts are then transmitted to a health center for a response. The user interfaces, data gathered, health setting, alerting mechanisms, health professionals and clinical interventions provided also varied widely. Three of the papers ${ }^{47-49}$ also reported the automated provision of tailored self-care advice to the patients experiencing sideeffects in addition to clinical interventions. A summary of the systems tested is shown in Table 1.

\section{Clinical outcomes}

The methodological designs, sample populations and sizes, as well as the outcomes measured, varied considerably and are summarized in Table 2 .

Kearney et al..$^{49}$ found that patients in the intervention group reported less fatigue post chemotherapy in a randomized controlled trial. However, there was no significant difference in reports of vomiting, nausea, diarrhea, sore mouth or throat between the two study arms. The authors also noted an unexplained decrease in prevalence, severity and distress caused by hand-foot syndrome in the control group.

Benefits seen in the intervention group of an RCT by Kroenke et $a .^{50}$ included lower severity of pain and greater improvement in depression across the study period. The intervention group also reported greater improvement in some secondary end points, including the Sheehan Disability Scale, individual domains of Health Related Quality of Life (HRQOL) measures, anxiety and physical symptom burden. Differences in self-reported disability days, physical health and overall quality of life were not statistically significant. Interestingly, this study

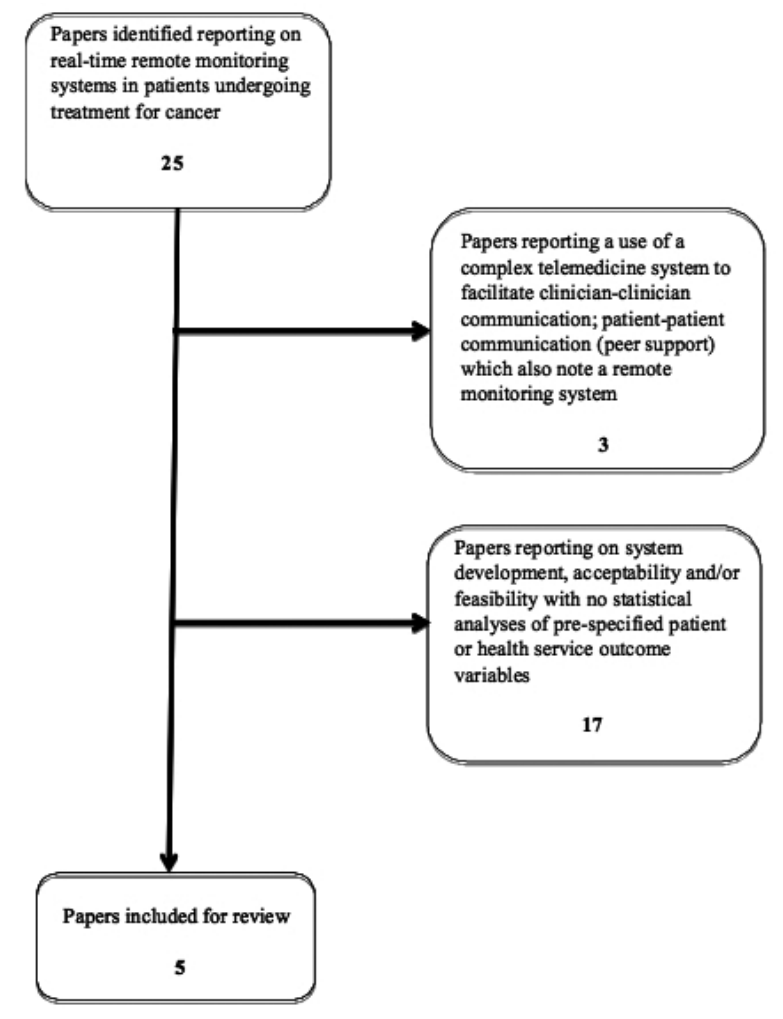

Figure 1. Summary of papers identified and subsequently excluded/included in this review. 


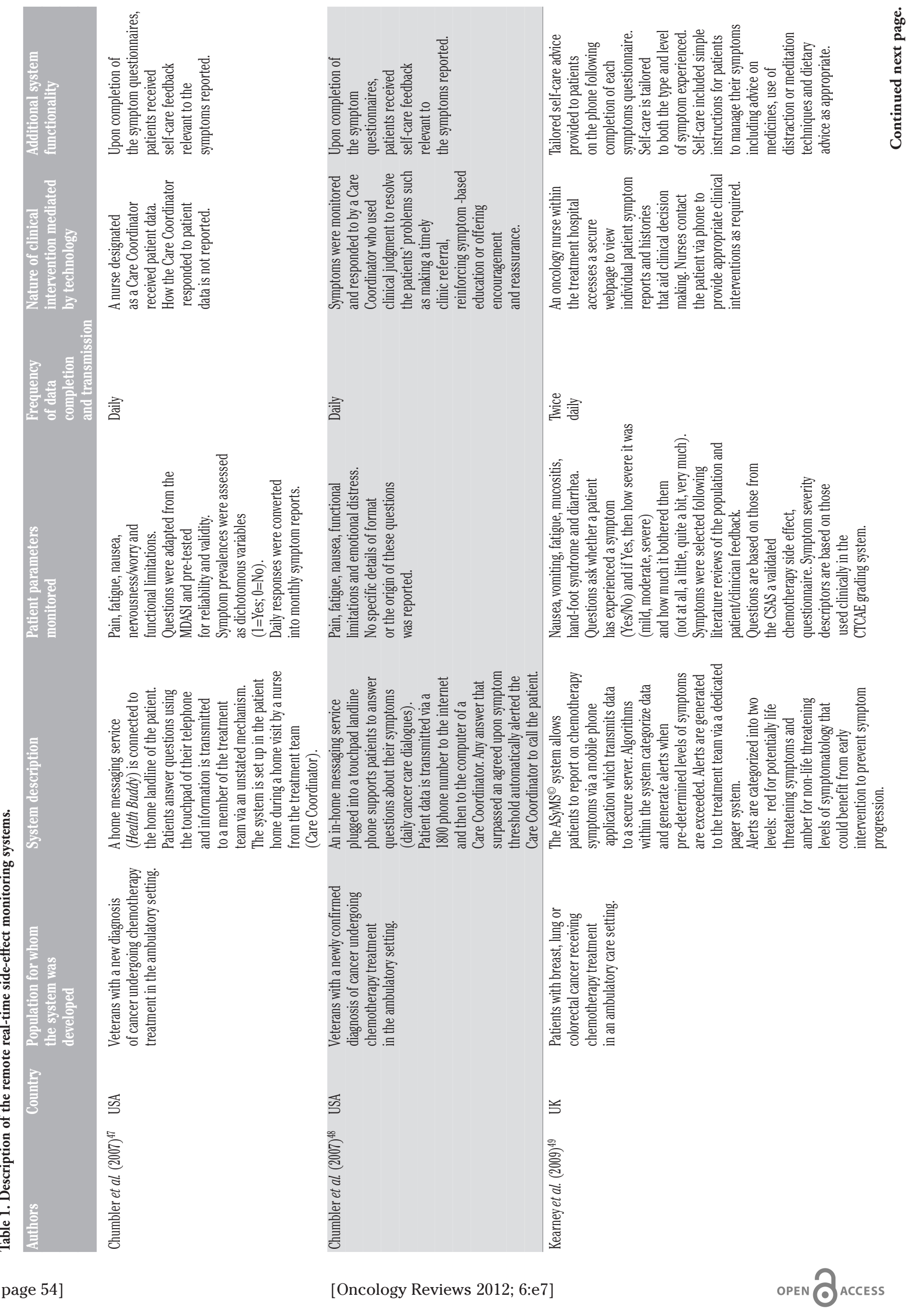




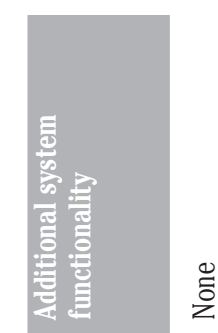

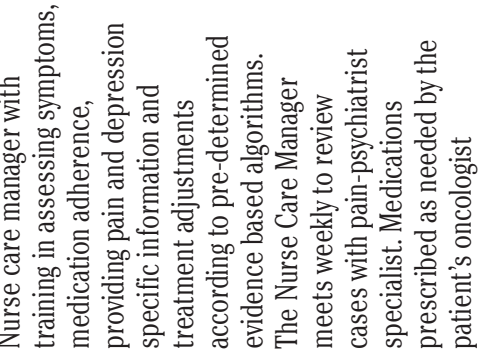

$\stackrel{0}{\check{0}}$

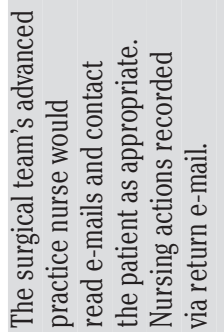

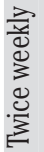

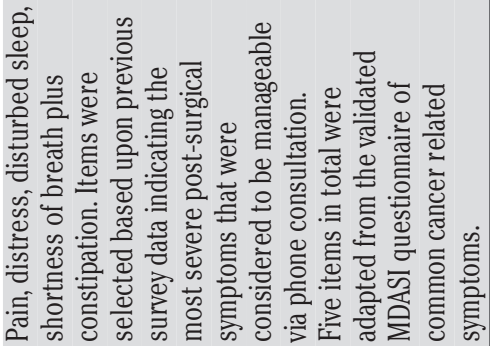

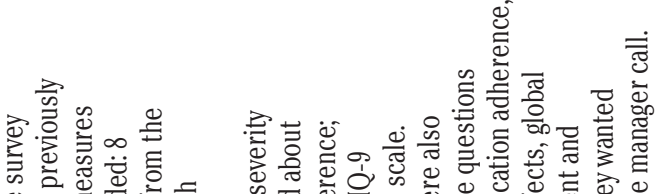

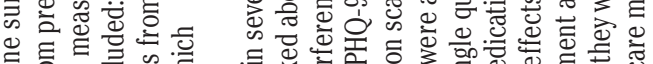

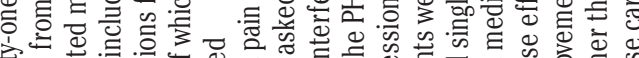

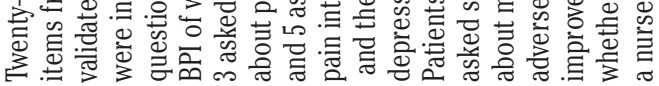

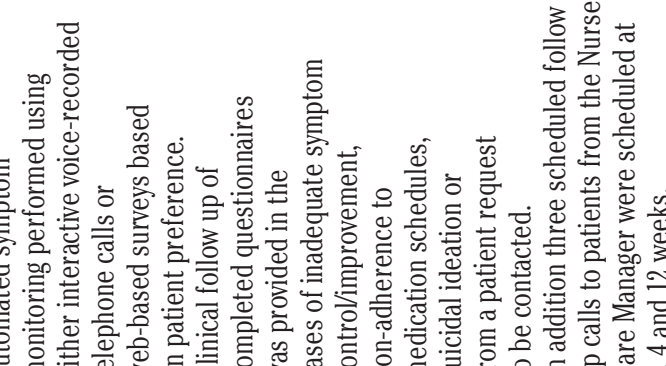

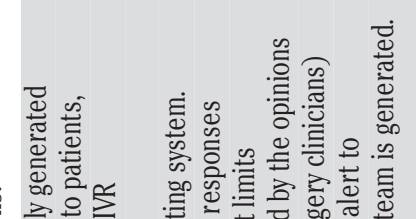

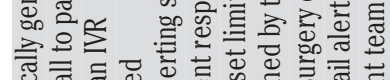

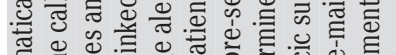

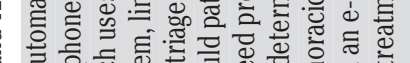

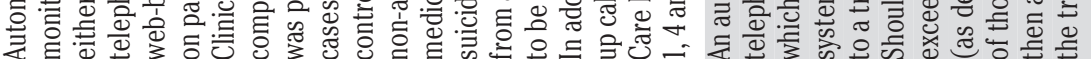

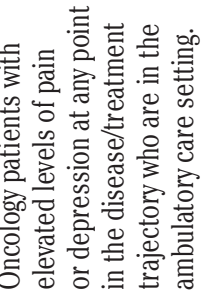

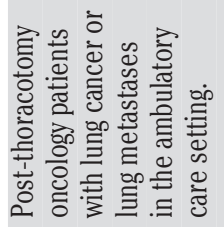

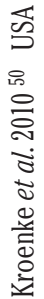

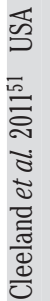



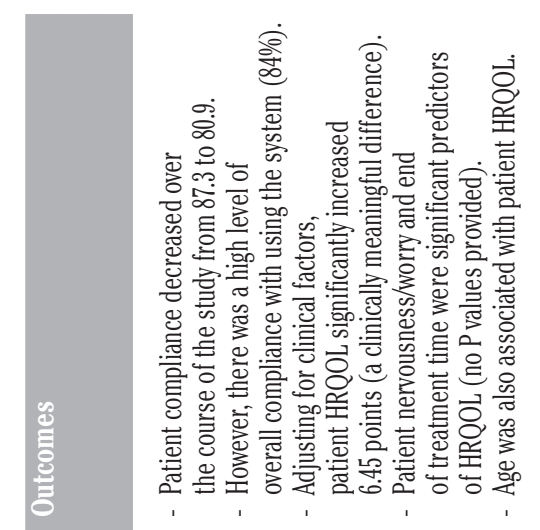

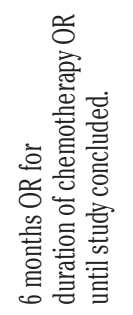

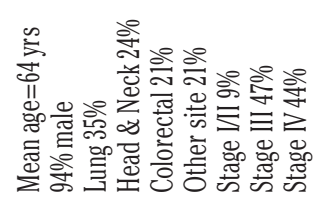

荧

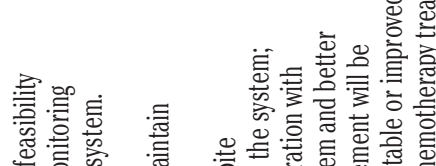

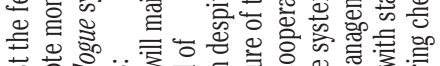

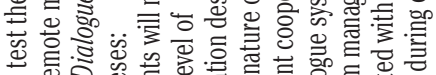

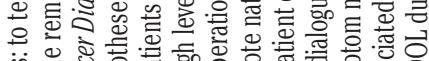

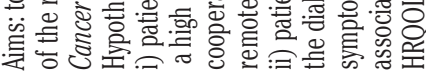

\section{:}

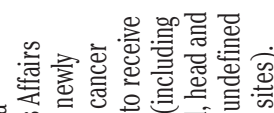

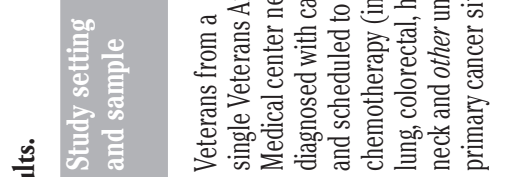

का

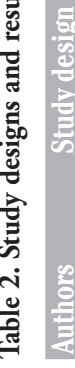

[page 56]

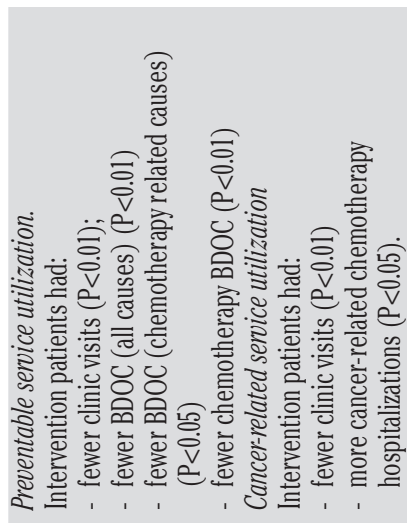

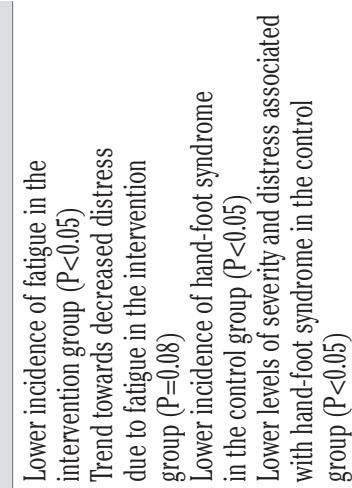

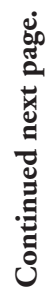

产

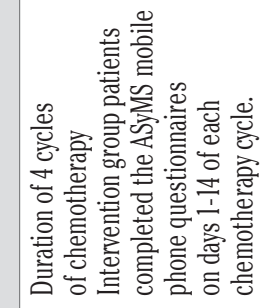

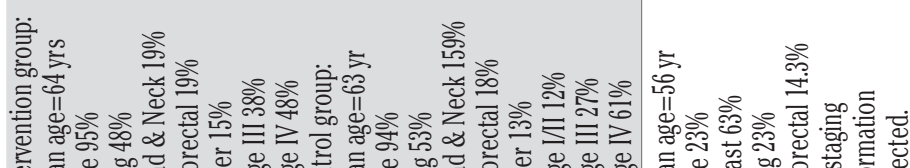

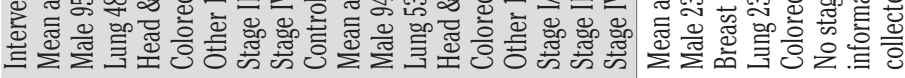

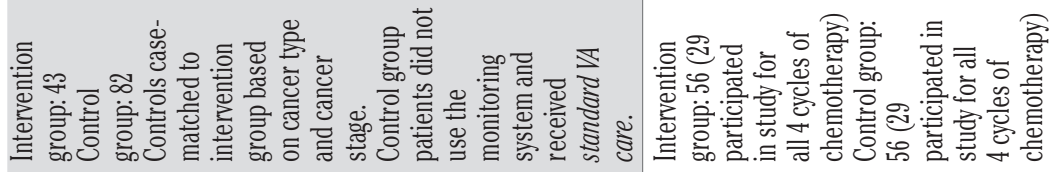

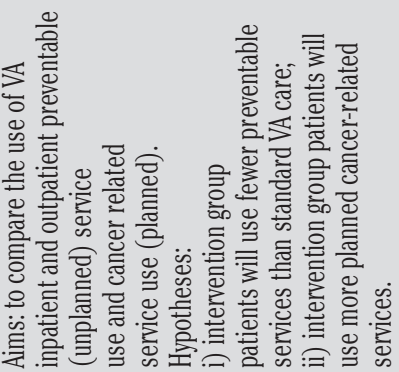

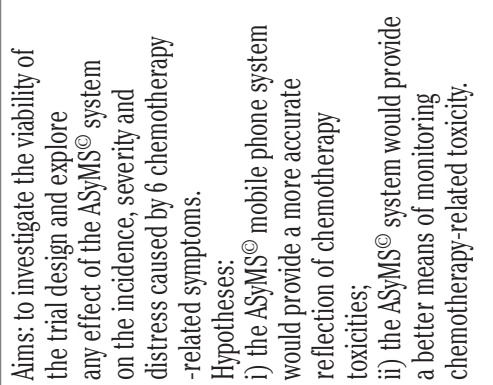
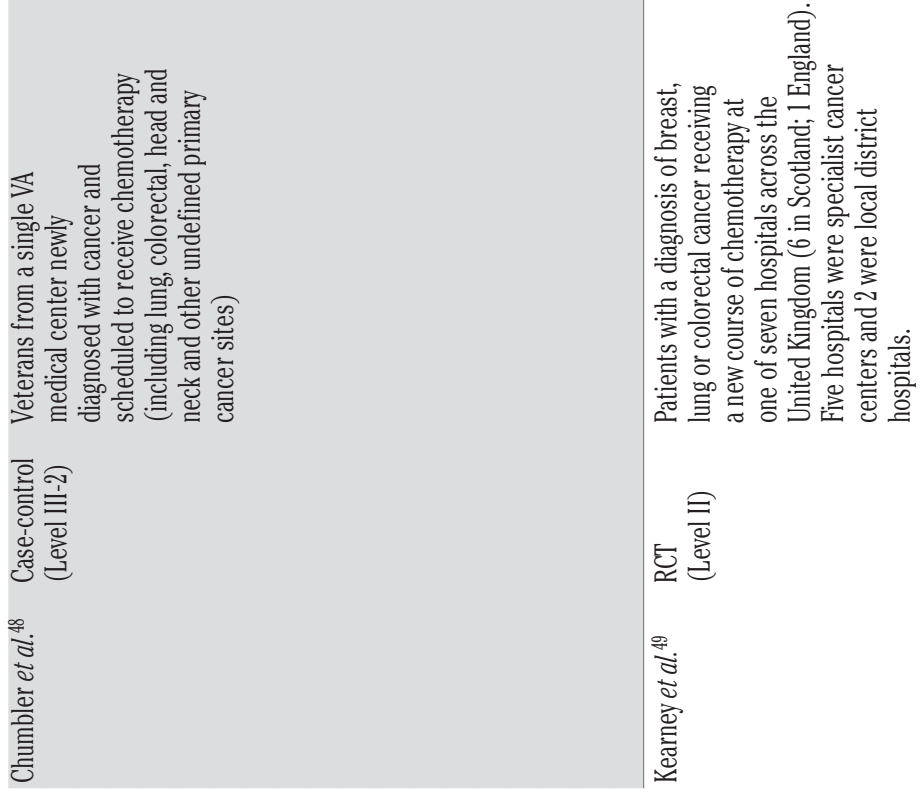

[Oncology Reviews 2012; 6:e7] 


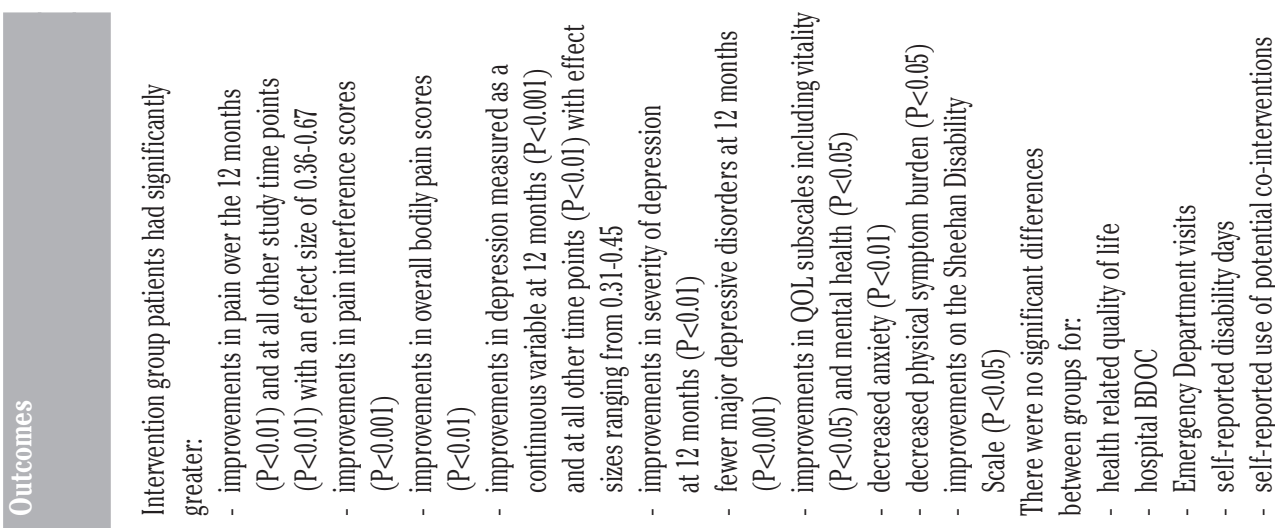

ن.

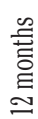

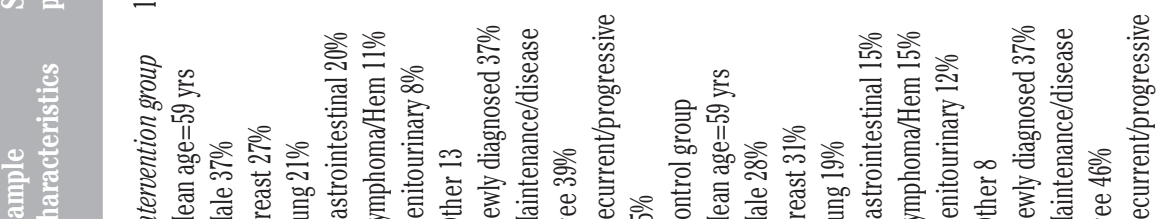

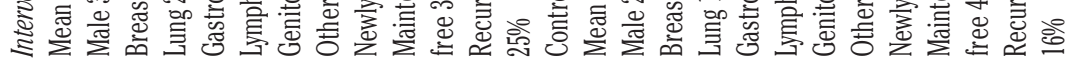

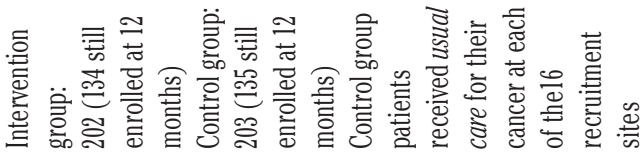
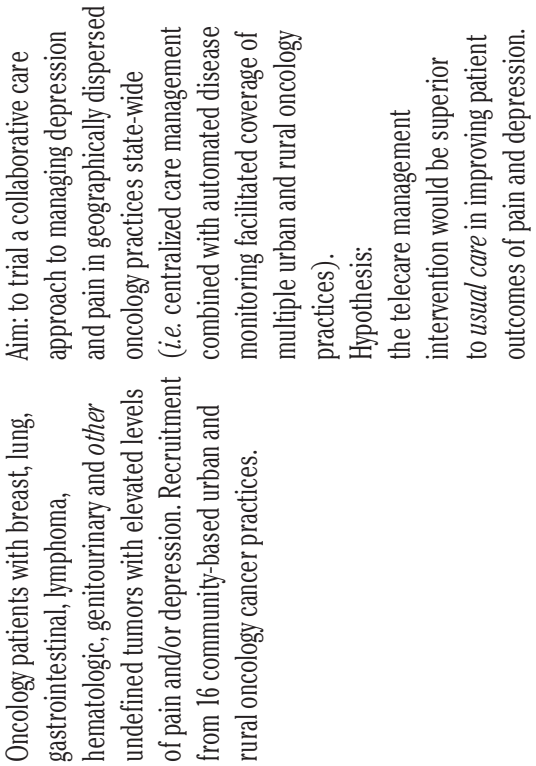

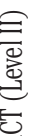




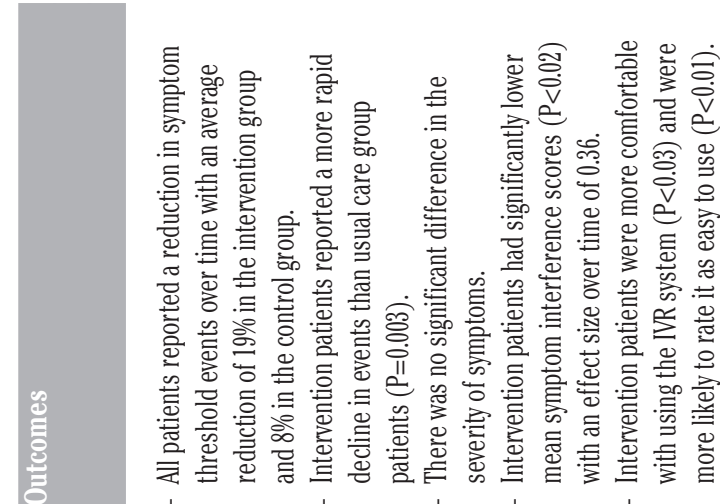

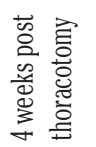

言站 产

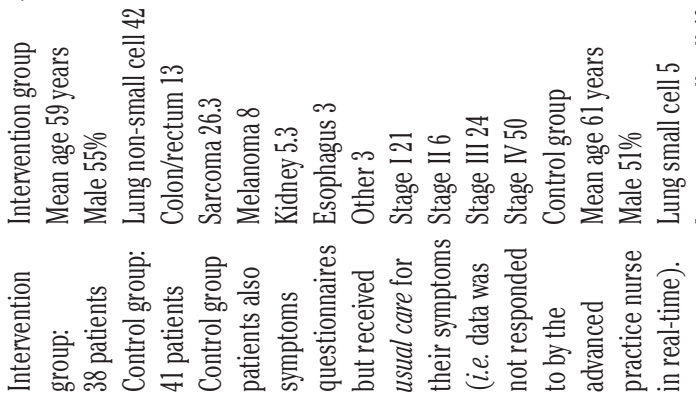

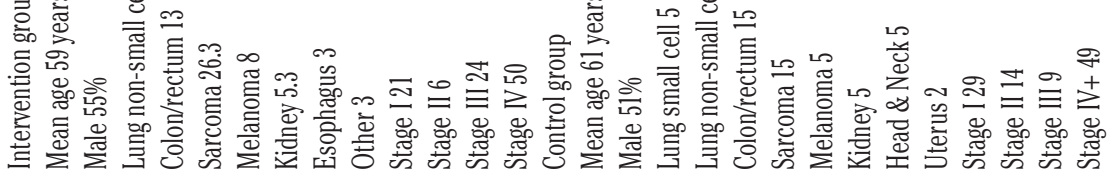
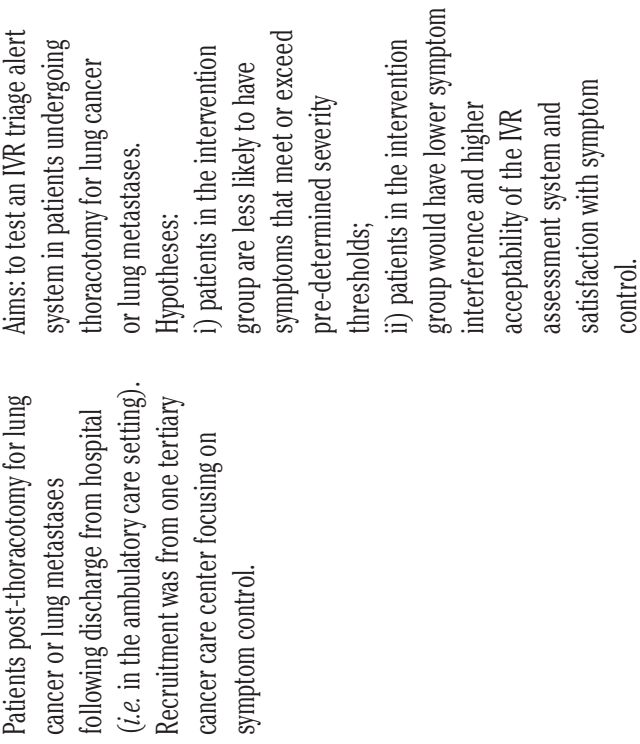


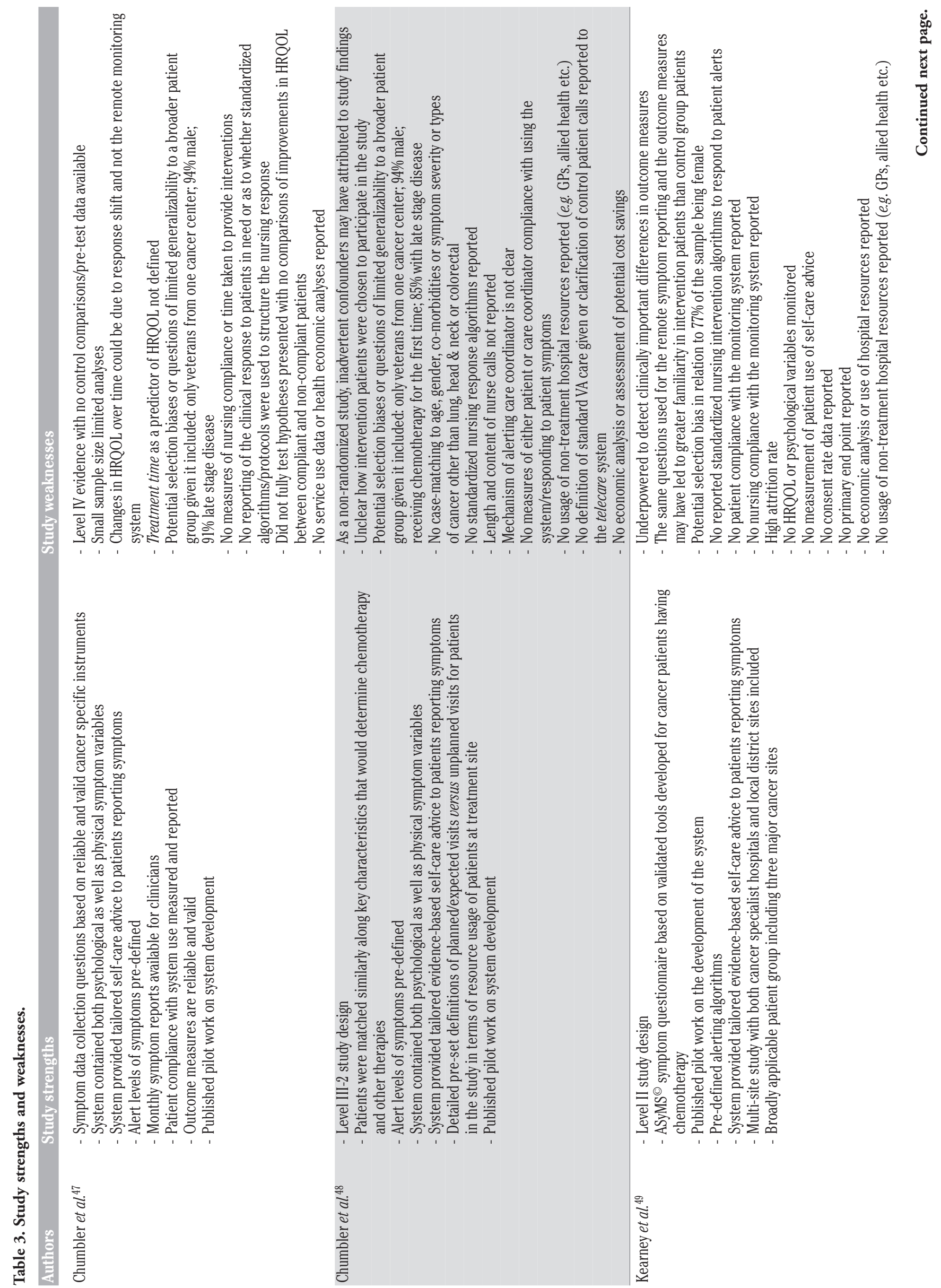



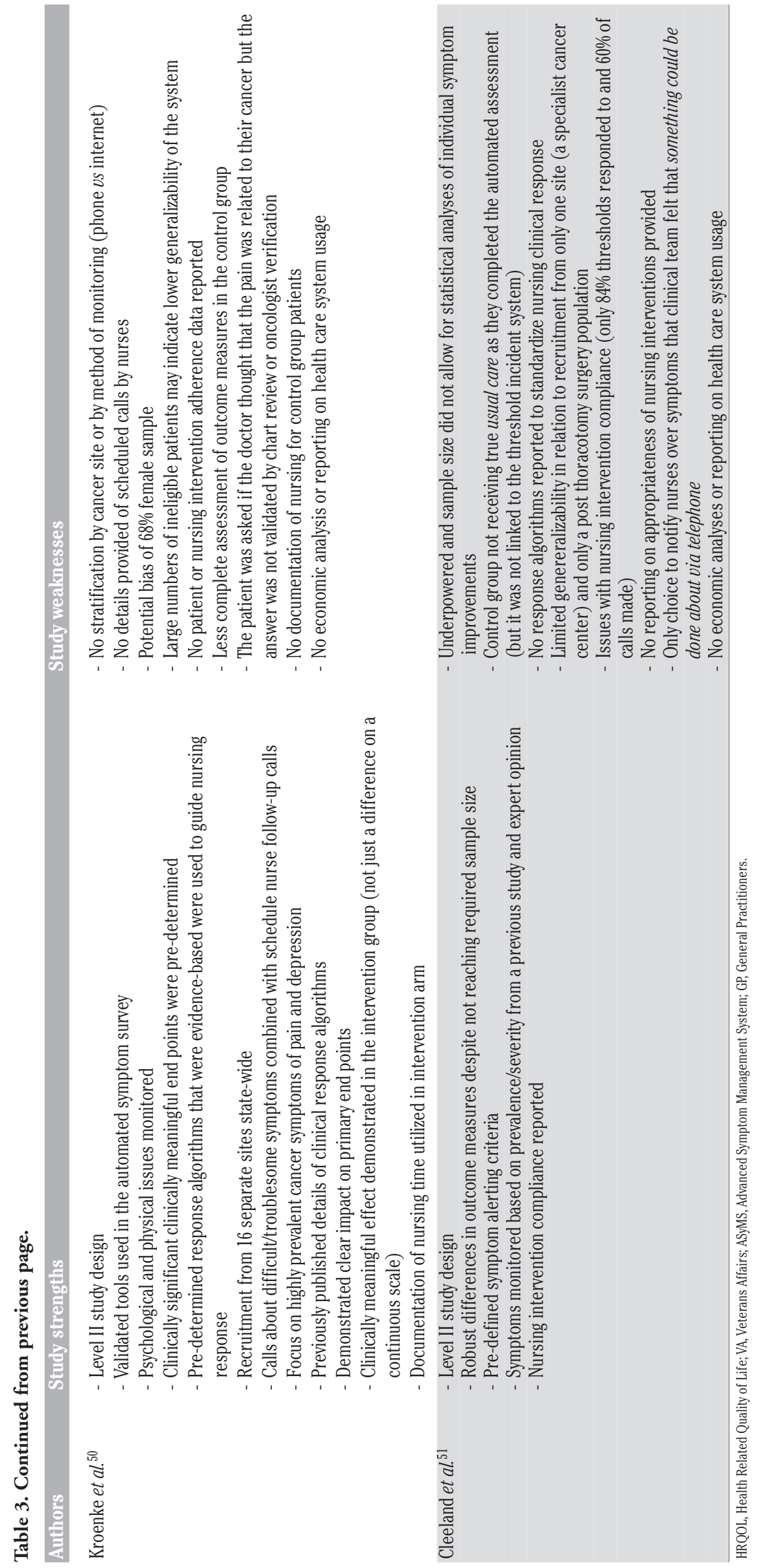

[page 60]

[Oncology Reviews 2012; 6:e7]

open $\bigcirc$ Access 
also had additional scheduled remote patient follow up by clinicians in addition to responding to remotely reported patient symptoms.

Cleeland et al. ${ }^{51}$ found that in their RCT of post-thoracotomy patients the intervention group had a significantly greater reduction in postoperative symptom threshold events; these had been pre-defined in consultation with thoracic surgery staff. The intervention group also saw a more rapid decline in numbers of events and had less interference with their activities of daily living. There were no statistically significant differences in self-reported severity of symptoms between the two study arms; however, the study was underpowered.

The post test case series performed by Chumbler et al. ${ }^{48}$ studied the effect on HRQOL in 34 patients utilising a home telehealth program. They demonstrated that HRQL in this small population increased over a 6 -month period. In the absence of a control group comparison, the possibility that this was due to response shift or another confounding variable cannot be ruled out.

\section{Healthcare system outcomes}

Only two papers reported on health care system outcomes. Building on their post test case series, Chumbler et al. ${ }^{47}$ performed a matched case-control study examining the impact of a remote symptom monitoring system on healthcare use among veterans undergoing chemotherapy. They demonstrated a decrease in the use of a number of preventable services but did not perform an economic analysis. In their study, Kroenke et al. ${ }^{50}$ did not observe any statistically significant difference in hospital days and emergency department visits between their two groups. No study performed any cost-benefit or other health economic analyses. This is possibly related to limitations in the medical record whereby all variables required to complete these complex assessments may not have been consistently registered.

\section{Study strengths and limitations}

A summary of the strengths and limitations is provided in Table 3. Comparisons of results between studies were limited by differences in design, outcomes measured, monitoring systems and methodologies used. Study limitations included small sample sizes (underpowered analyses), participant selection bias, population generalizability and low levels of evidence linked to study design. ${ }^{46}$ However, despite the limitations, significant differences were observed in RCTs and the improvements and changes reported in patients were generally robust.

\section{Discussion}

To date there have been few studies in the cancer population of the impact of telecommunication-based devices that regularly collect data from patients at home and transmit the results to a healthcare professional for response in real-time. Given the distressing and serious sideeffects that are common during and following cancer treatments ${ }^{2-21}$ this is surprising given the apparent cost-effectiveness of these systems in chronic disease patients. This review aimed to identify papers that not only described remote monitoring systems in cancer patients, but also statistically analyzed patient clinical and health care system outcomes. Only five published trials were found, of which three were RCTs and of these only one was sufficiently powered. No studies were completed within the context of the Australian health care system.

The majority of the studies had several methodological limitations which include using the same questionnaire for the intervention and outcome measure, ${ }^{49}$ heterogeneous populations precluding more precise estimates of treatment effect in specific types or stages of cancer, ${ }^{47-50}$ less complete assessment of outcome measures in the control group,$^{50}$ eligibility criteria requiring the presence of the symptom at trial commencement ${ }^{50}$ and the control group using the automated system without response such that the control group was in effect no longer receiving usual care..$^{51}$ These limitations together with the differences in remote monitoring systems and patient populations reduced our ability to generalize or confirm the findings of previous trials and to make cross-study comparisons. Essentially, the available RCTs have examined three separate complex interventions and it is practically impossible to make a comparison between them. It is also difficult to know whether changes in patient and health system outcomes are due to the symptoms monitored, the frequency of monitoring, the technological interface, the person and/or algorithms guiding responses to the alerts, or whether or not automatic self-care advice is given.

Despite the limitations, there have been some promising results to guide future research. Of note, the adequately powered study by Kroenke et al. ${ }^{50}$ demonstrated clinically significant intervention effects on the primary end points (pain and depression) as well as other secondary outcomes. It must be remembered, however, that this study was conducted in patients with pre-existing pain or depression so the results may not be generalized to those without a pre-existing condition. Although the study by Cleeland et al. ${ }^{51}$ was underpowered, it did demonstrate large between-group differences in post-thoracotomy symptom threshold events overall, and larger future studies may be able to identify differences within individual symptoms and severity. Remote symptom monitoring systems may reduce fatigue post chemotherapy ${ }^{49}$ Fatigue is a common and often overlooked side-effect which is difficult to manage and is linked with poor HRQOL and mood disturbance.

It is interesting that while reassurance was a key theme that emerged from the previous qualitative studies, none of the RCTs in this review included anxiety as a primary outcome measure. Kearney $e t$ $a l .{ }^{49}$ did examine the distress associated with each of the side-effects and the differences between the two groups; however, they did not include a measure of overall anxiety. The study by Kroenke et al. ${ }^{50}$ was the only study to measure anxiety as a secondary outcome and found that it was significantly reduced in the intervention monitoring group. Alongside improvements in depression, this suggests that remote monitoring shows promise for improving patient psychological as well as physical outcomes.

Despite the fact that patients were monitored less often and were not provided with automated self-care advice, the results from the study by Kroenke et al. ${ }^{50}$ are the most convincing evidence for the effectiveness of remote monitoring in a wide-variety of cancer patients. This system differs from the others in that a team of people (nurse, psychiatrist and oncologist) are involved in the management of the symptoms reported in a structured and consistent manner, rather than the responsibility falling on one person. This may have influenced the quality of the intervention and hence the outcome of the trial. In addition, interventions were guided by previously published evidence-based clinical algorithms (including pharmacotherapy) that were not reported in the other studies. This system and associated intervention also differed from all of the others in that scheduled remote follow ups with patients were completed in addition to responding ad hoc to problematic symptoms. The contribution that this may have made to the overall study results is unclear. The success of this study may just be that it is easier to show improvements in pre-existing symptoms when compared with aiming to prevent symptoms or future side-effects.

No study clearly documented both nurse and patient adherence to the use of the remote monitoring systems or reported on the appropriateness of actions taken or whether they were consistent with any predefined clinical response algorithms. Those systems providing patients with self-care advice were also unable to report on the frequency of 
patient access to this information, or more importantly, whether the patients used the information provided. Cleeland et al ${ }^{51}$ reported that only a subset of threshold events triggered clinician responses which may have also affected study outcomes. Kearney et al. ${ }^{49}$ provided very little information about clinical interventions instituted in response to alerts so it is difficult to tell whether or not they were consistent across the study and between study sites.

Although not randomized, the case-control study by Chumbler et al.48 demonstrated promising benefits to the healthcare system in terms of unplanned presentations and bed days of care. Given that the introduction of a new technology has a price attached to it, there is a need to establish that this cost is offset by the clinical impact of using these devices. No health economic analyses of remote real-time monitoring devices in cancer were identified in the peer-reviewed literature. These studies are necessary if there is to be widespread uptake of this type of technology as healthcare administrators are unlikely to fund an intervention that does not have economic benefit. It is important to remember that technology such as remote monitoring does not replace face-to-face patient contact but complements it. As such, there are costs associated with the provision of the technology for patient use, data transfer charges and time taken for the health professional to monitor and respond to such systems. Whether these then decrease health care system costs across the board is a broader issue. Not only is it important to measure inpatient and outpatient costs and health care system usage, it is also necessary to consider other costs that may be incurred by the patient from the implementation of these models of care, for example, visits to general practitioners or other community health services. Newer technology being developed may also mean that additional parameters could be measured in the ambulatory care setting when needed (e.g. blood tests) and the costs of devices to allow this also need to be factored into future cost analyses.

All of the studies included in this review have been published in the past five years which suggests that this is an emerging facet of cancer care. However, in addition to the completion of more rigorous studies to fully assess cost-effectiveness of such remote monitoring systems, health services also need to think about how such care provision can be funded given the limitations of current systems. It is also possible that such remote monitoring will not prove cost-effective for all patients undergoing treatment. The successful study of Kroenke et al..$^{50}$ only looked at patients who already had significant clinical issues. It is, therefore, possible that such systems may only be cost-effective for those cancer patients who are at high risk of adverse outcomes due to high toxicity treatments (e.g. hematological cancer patients), already symptomatic or who have significant co-morbidities. Future research should also focus on these groups when considering cost-benefits of remote monitoring systems.

\section{Conclusions and future directions}

There are very few studies examining the outcomes of real-time remote symptom monitoring devices in patients with cancer. There is some evidence that they can reduce morbidity and prevent unplanned hospitalizations. However, results are mixed. It is very difficult to make generalizations given that there is no consistency in the monitoring devices used, disease type, treatments, side-effects or symptoms monitored, and breadth of management and interventions. More sufficiently powered randomized control trials, which also assess adherence to the intervention and include a full economic analysis, are warranted. A full examination of these benefits is a key priority within the Australian health care system alongside an exploration of health care funding models to support their implementation. With the aging of the population, and the resultant increase in cancer diagnoses, it is vital to explore systems to provide ambulatory care in a safer and more economically viable manner as well as to potentially target high-risk groups who would benefit most from such interventions.

\section{References}

1. Australian Institute of Health and Welfare \& Australasian Association of Cancer Registries 2010. Cancer in Australia: in brief 2010. Cancer series no. 59, 2010; Cat. No. CAN 55. Canberra: AIHW.

2. Hesketh PJ, Sanz-Altamira P, Bushey J, Hesketh AM. Prospective evaluation of the incidence of delayed nausea and vomiting in patients with colorectal cancer receiving oxaliplatin-based chemotherapy. Support Care Cancer 2012;5;1043-7.

3. Jones JM, Qin R, Bardia A, et al. Antiemetics for chemotherapyinduced nausea and vomiting occurring despite prophylactic antiemetic therapy. J Pall Med 2011;14:810-4.

4. Blanke CD, Bot BM, Thoman DM, et al. Impact of young age on treatment efficacy and safety in advanced colorectal cancer: a pooled analysis of patients from Nine First-Line Phase III Chemotherapy Trials. J Clin Onc 2011;29:2781-6.

5. Ridolfi L, Bertetto 0, Santo A, et al. Chemotherapy with or without low-dose interleukin-2 in advanced non-small cell lung cancer: results from a phase III randomized multicentric trial. Int J Onc 2011;39:1011-7.

6. Madarnas Y, Dent SF, Husain SF, et al. Real-world experience with adjuvant fec-d chemotherapy in four Ontario regional cancer centres. Curr Onc 2011;18:119-25.

7. Lanoix, JP, Pluquet E, Lescure FX, et al. Bacterial infection profiles in lung cancer patients with febrile neutropenia. BMC Infect Dis 2011;11:183.

8. Kuderer NM, Dale DC, Crawford J. Mortality, morbidity, and cost associated with febrile neutropenia in adult cancer patients. Cancer 2006;106:2258-66.

9. Park VJ, Trovoto JA. Frequency of nausea and vomiting after chemotherapy. Am J Health Syst Pharm 2004;61:722-3.

10. Du XL, Osborne C, Goodwin JS. Population-based assessment of hospitalizations for toxicity from chemotherapy in older women with breast cancer. J Clin Oncol 2002;20:4636-42.

11. Richardson A, Ream E. The experience of fatigue and other symptoms in patients receiving chemotherapy. Eur J Cancer Care 1996;5:24-30.

12. Scully C, Sonis S, Diz PD. Oral mucositis. Oral Dis 2006;12:229-41.

13. Sonis S, Oster G, Fuchs H. Oral mucositis and the clinical and economic outcomes of haematopoietic stem-cell transplantation. J. Clin. Oncol 2001;19:2201-5.

14. Glaus A. Assessment of fatigue in cancer and non-cancer patients and in health individuals. Support Care Cancer 1993;1:305-15.

15. Fransson P. Fatigue in prostate cancer patients treated with external beam radiotherapy: a prospective 5 -year long-term patientreported evaluation. J Cancer Res Ther 2010;6:516-20.

16. Henson C. Chronic radiation proctitis: issues surrounding delayed bowel dysfunction post-pelvic radiotherapy and an update on medical treatment. Therap Adv Gastroenterol 2010;3:359-65.

17. Shapiro CL, Recht A. Side effects of adjuvant treatment of breast cancer. N Engl J Med 2001;344:1997-2008.

18. Zachariah B, Balducci L, Venkattaramanabalaji GV, et al. Radiotherpy for cancer pateints aged 80 and lder: a study of effectiveness and side-effects. Int J Rad Onc Biol Phys 1997;39:1125-9.

19. Trotti A, Belim LA, Epstein JB, et al. Mucositis incidence, severity and associated outcomes in patients with head and neck cancer receiving radiotherapy with or without chemotherapy: a systematic literature review. Rad Onc 2003;66:253-62. 
20. Katz J, Poleshuck EL, Andrus CH, et al. Risk factors for acute pain and its persistence following breast cancer surgery. Pain 2005;119:16-25.

21. Breen SJ, Baravelli CM, Schofield PE, et al. Is symptom burden a predictor of anxiety and depression in patients with cancer about to commence chemotherapy? Med J Aust 2009;190:586-9.

22. Barnett T, Chumbler N, Vogel W. The effectiveness of a care coordination home telehealth program for veterans with diabetes mellitus: a 2-year follow-up. Am J Manag Care 2006;12:4467-74.

23. Chumbler N, Neugaard B, Kobb R. Evaluation of a care coordination/home-telehealth program for veterans with diabetes. Eval Health Prof 2005;28:464-78.

24. Rasmussen LM, Phanareth K, Nolte H, Backer V. Internet-based monitoring of asthma: a long-term, randomized clinical study of 300 asthmatic subjects. J All Clin Immunol 2005;115:1137-42.

25. Louis A, Turner T, Gretton M, et al. A systematic review of tele-monitoring for the management of heart failure. Eur J Heart Fail 2003;5:583-90.

26. Bartoli L, Zanaboni P, Masella C, Ursini N. Systematic review of telemedicine services for patients affected by chronic obstructive pulmonary disease (COPD). Telemed J E Health 2009;15:877-83.

27. Santamaria N, Carville K, Ellis I, Prentice J. The effectiveness of digital imaging and remote wound consultation on healing rates in chronic lower leg ulcers in the Kimberley region of Western Australia. Prim Intent 2004;12:62-70.

28. Noel HC, Vogel DC, Erdos JJ, et al. Home telehealth reduces healthcare costs. Telemed J E Health 2004;10:170-83.

29. Cleeland JGF, Louis AA, Rigby AS, et al. Noninvasive home telemonitoring for patients with heart failure at high risk of recurrent admission and death: the Trans-European Network-Home-Care Management System (TEN-HMS) study. J Am Coll Cardiol $2005 ; 45: 1654-64$

30. Kearney N, Miller M, Maguire R, et al. WISECARE+: Results of a European study of a nursing intervention for the management of chemotherapy-related symptoms. Euro J Onc Nurs 2008;12:443-8.

31. Mooney K, Beck S, Friedman R, Farzanfar R. Telephone-linked care for cancer symptom monitoring, a pilot study. Cancer Pract 2002;10:147-54

32. Lind L, Karlsson D. A system for symptom assessment in advanced palliative home healthcare using digital pens. Med Inform 2004;29:199-210.

33. Lind L, Karlsson D, Fridlund B. Digital pens and pain diaries in palliative home healthcare: professional caregivers experience. Med Inform Internet Med 2007;32:287-96.

34. Lind L, Karlsson D, Fridlund B. Patients' use of digital pens for pain assessment in advanced palliative home healthcare. Int $\mathrm{J}$ Med Inform 2008;77:129-136.

35. McGee MR Gray P. A handheld chemotherapy symptom management system: results from a preliminary outpatient field trial. Health Info J 2005;11:243-58.

36. Kearney N, Kidd L, Miller M, et al. Utilising handheld computers to monitor and support patients receiving chemotherapy: results of a UK based feasibility study. Support Care Cancer 2006;14:742-52.
37. Maguire R, Miller M, Sage M, et al. Results of a UK based pilot study of a mobile phone based advanced symptom management system (ASyMS $($ ) ) in the remote monitoring of chemotherapy related toxicity. Clin Effect Nurs 2005;9:202-10.

38. Maguire R, McCann L, Miller M, Kearney N. Nurse's perceptions and experiences of using of a mobile-phone-based Advanced Symptom Management System (ASyMS $\odot$ ) to monitor and manage chemotherapy-related toxicity. Eur J Onc Nurs 2008;12:380-6.

39. McCann L, Maguire R, Miller M, Kearney K. Patients' perceptions and experiences of using a mobile phone-based advanced symptom management system (ASyMS (C) to monitor and manage chemotherapy related toxicity. Eur J Cancer Care 2009;18:156-64.

40. McCall K, Keen J, Farrer K, et al. Perceptions of the use of a remote monitoring system in patients receiving palliative care at home. Int J Pall Nurs 2008;14:426-31.

41. Gibson F, Aldiss S, Taylor RM, et al. Involving health professionals in the development of an advanced symptom management system for young people: the ASyMS-YG study. Eur J Onc Nurs 2009;13:187 92.

42. Gibson F, Aldiss S, Taylor RM, et al. Utilization of the Medical Research Council evaluation framework in the development of technology for symptom management: the ASyMS-YG $\odot$ Study. Cancer Nurs 2010;33:343-52.

43. Weaver A, Young AM, Rowntree J, et al. Application of mobile phone technology for managing chemotherapy-associated sideeffects. Ann Oncol 2007;18:1887-92.

44. Head BA, Studts JL, Bumpous JM, et al. Development of a telehealth intervention for head and neck cancer patients. Telemed J E-Health 2009;15:44-52.

45. Head BA, Keeney C, Studts JL, et al. Feasibillity and acceptance of a telehealth intervention to promote symptom management during treatment for head and neck cancer. J Support Oncol 2011;9:e1-11.

46. Merlin T, Weston A, Tooher R. Extending an evidence hierachy to include topics other than treatment: revising Australian levels of evidence. BMC Med Res Meth 2009;9:34-41.

47. Chumbler NR, Mkanta WN, Richardson LC, et al. Remote patientprovider communication and quality of life: empirical test of a dialogic model of cancer care. J Telemed Telecare 2007;13:20-5.

48. Chumbler NR, Kobb R, Harris L, et al. Healthcare utilization among veterans undergoing chemotherapy: the impact of a cancer care coordination/home-telehealth program. J Amb Care Manage 2007;30:308-17.

49. Kearney N, McCann L, Norrie J, et al. Evaluation of a mobile phonebased, advanced symptom management system (ASyMS) in the management of chemotherapy-related toxicity. Support Care Cancer 2009;17:437-44.

50. Kroenke K, Theobald D, Wu J, et al. Effect of telecare management on pain and depression in patients with cancer: a randomized trial. JAMA 2010;304:163-71.

51. Cleeland CS, Wang XS, Shi Q, et al. Automated symptom alerts reduce postoperative symptom severity after cancer surgery: a randomized controlled clinical trial. J Clin Onc 2011;29:994-1000. 